\title{
An Application of a 3-DOF Parallel Manipulator for Earthquake Simulations
}

\author{
Marco Ceccarelli, Erika Ottaviano, Gianni Castelli
}

\begin{abstract}
In this paper a novel application is presented for a parallel manipulator to be used as an earthquake motion simulator. A formulation and experimental results are presented for a 3-DOF CaPaMan (Cassino Parallel Manipulator) prototype to simulate point seismograms and 3D earthquake motion. The rigid body acceleration (linear acceleration, angular velocity and acceleration) has been experimentally evaluated to simulate real 3D earthquakes by using the parallel manipulator.
\end{abstract}

Index Terms - Parallel Manipulators; Experimental Robotics; Earthquake Simulators

\section{INTRODUCTION}

Nowadays it is of great interest to be able to study earthquakes, since their effects may cause loss of life and high cost to replace and repair what has been destroyed. Therefore, motions that are commonly associated with earthquakes cab be reproduced by earthquake simulators used in laboratories of Civil Engineering Departments, like for example those referred in [1]-[7]. Those simulators are designed and constructed to mimic these cataclysmic events; studying the effects of earthquakes on buildings; and consequently developing advanced technologies for protecting buildings from big earthquakes.

Several types of earthquake simulators are available but most of them do not take into account and operate for a $3 \mathrm{D}$ motion of the terrain due to the earthquake. In fact, usually seismograms that are available from seismic sites concern with the characteristics of point motion by using accelerometers.

Nevertheless, the 3D motion of the terrain can strongly affect the dynamic excitation of the buildings. Therefore, recently during the 1997 earthquakes in center Italy, novel attention has been addressed to the $3 \mathrm{D}$ aspects of earthquake motion by installing proper systems to acquire and elaborate data, as explained in [8]-[10].

At LARM: Laboratory of Robotics and Mechatronics, a spatial parallel manipulator has been used with a proper formulation and suitable sensorization for simulating earthquake motion.

In particular, the 3D motion capability of CaPaMan parallel architecture has been used to simulate 3D earthquake motion through an extension of previous experiences, [12],

Erika Ottaviano and Marco Ceccarelli are with LARM: Laboratory of Robotics and Mechatronics at DiMSAT - University of Cassino, 03043 Cassino (Fr), Italy (corresponding author Erika Ottaviano phone: +39-07762993665; fax: +39-0776-2993711; e-mail: ottaviano/ceccarelli@unicas.it).
[13]. A 3D motion for the terrain body can be considered as a novelty for earthquake simulators. In this paper, results are reported for a study based on experimental activity by using CaPaMan architecture as an earthquake simulator of general seismic motion.

\section{EARTHQUAKE CHARACTERISTICS}

A seismic motion can be described as function of chemical and physical characteristics of the terrain, which can be considered as a composite body with several stratified masses, [14]. In order to study a seismic motion one can identify the period of a seismic cycle and characteristic length for each seismic wave. Seismic waves can be grouped into 3 main types: the compression expansion waves $\mathrm{P}$, transversal waves $\mathrm{S}$, and superficial waves $\mathrm{M}$. They can be classified by referring both to the propagation speed and terrain movements, as shown in Fig. 1. The $\mathrm{P}$ waves propagate through a spring-like-motion with a typical period between 0.1 and $0.2 \mathrm{sec}$; the $\mathrm{S}$ waves refer to sea-like waves with a typical period between 0.5 and $1 \mathrm{sec}$; the $\mathrm{M}$ waves involve only the terrain surface with a typical period between $20 \mathrm{sec}$ and $1 \mathrm{~min}$. The intensity, shape and duration of a seismic motion can depend also on the characteristics of the terrain through which the seismic waves propagate. In Fig. 1c) the main difference is represented among the above mentioned seismic waves in terms of acceleration magnitude and characteristic period of oscillating motion. This oscillation motion is responsible of a periodical excitation of structures that can be damaged when resonance situation occurs. Usually, critical resonant condition can be analyzed in terms of translational seismic components, but even angular motion can strongly contribute to the resonant excitation.

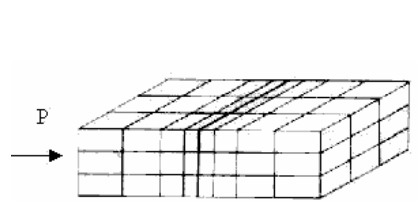

a)

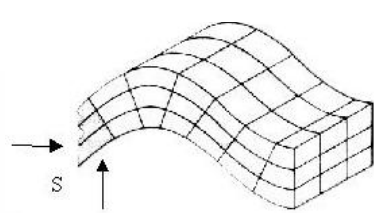

b)

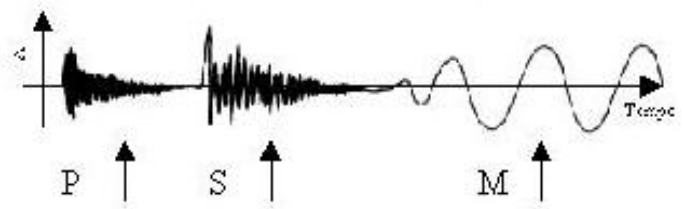

c)

Figure 1. Basic characteristics of seismic waves: a) compression and expansion waves; b) transversal waves; c) types of seismograms. 
Earthquake evaluation is performed by monitoring the basic characteristics of a seismic motion, mainly in terms of acceleration magnitude and time history [14], as shown in Fig. 1c). However, even displacement and velocity of the wave motion are of great interest. In addition, 3D motion evaluation can be important in order to consider the wave space motion and its propagation on the terrain surface.

\section{EARTHQUAKE 3D SEISMIC Motion}

According to the authors' knowledge, the first data for investigation on 3D motion of terrain under earthquake effects has been collected by the Italian Seismic Service (SSN) in occasion of the earthquake events in central Italy in 1997-'98, as reported in $[8 ; 10]$.

The 3D motion effects on resistant constructions are well known, and in fact suitable dynamic analyses and designs are current practice for constructions.

A 3D terrain motion during a seismic event can be considered as related to angular motion due to seismic wave propagation. It can be recognized basically by looking at the displacement of terrain elements in the sketches of Fig. 1.

In order to evaluate $3 \mathrm{D}$ seismic motion of terrain element, one can define a flat portion and consider it as a rigid plane at which a Cartesian frame can be attached.

In order to completely define the rigid body acceleration (linear acceleration, angular velocity and acceleration) it is necessary to know the linear acceleration ( $\mathrm{a}_{\mathrm{P} 0 \mathrm{x}}, \mathrm{a}_{\mathrm{P} 0 \mathrm{y}}, \mathrm{a}_{\mathrm{P} 0 \mathrm{z}}$ ), of one point $\mathrm{P}_{0}$ belonging to the rigid body angular velocity $\left(\omega_{\mathrm{x}}\right.$, $\left.\omega_{\mathrm{y}}, \omega_{\mathrm{z}}\right)$ and angular acceleration $\left(\dot{\omega}_{\mathrm{x}}, \dot{\omega}_{\mathrm{y}}, \dot{\omega}_{\mathrm{z}}\right)$, with respect to a frame attached to the body and with the origin coincident with $\mathrm{P}_{0}$. Indeed, the above-mentioned nine parameters are necessary and sufficient to completely describe the acceleration of a rigid body.

A $3 \mathrm{D}$ motion is known when the Kinematics is evaluated for a point and Cartesian frame, or alternatively for three points or four coplanar points, [15].

Figure 2 shows practical solution that SSN has implemented to install several seismograph accelerometers on the basement floor Stallone (room) at Assisi Convent, [13].

In the case of Fig. 2 points $\mathrm{P}_{0}, \mathrm{P}_{1}, \mathrm{P}_{2}$, and $\mathrm{P}_{3}$ have been defined as laying on the floor plane. Thus, the angular motion of the reference floor plane with respect to the fixed frame $\mathrm{P}_{0^{-}}$ $\mathrm{XYZ}$ can be expressed by the angular velocity components, and acceleration components, as functions of the acceleration components of points $\mathrm{P}_{0}, \mathrm{P}_{1}, \mathrm{P}_{2}$, and $\mathrm{P}_{3}$ from the expressions

$$
\begin{aligned}
& a_{P_{1 X}}=a_{P_{0 X}}+\omega_{Y}\left(\omega_{X} b+\omega_{Y} a\right)+\omega_{Z}^{2} a-\dot{\omega}_{Z} b \\
& a_{P_{1 Y}}=a_{P_{0 Y}}-\omega_{Z}^{2} b-\omega_{X}\left(\omega_{X} b+\omega_{Y} a\right)-\dot{\omega}_{Z} a \\
& a_{P_{1 Z}}=a_{P_{0 Z}}-\omega_{X} \omega_{Z} a+\omega_{Y} \omega_{Z} b+\dot{\omega}_{X} b+\dot{\omega}_{Y} a \\
& a_{P_{2 Y}}=a_{P_{0 Y}}-\omega_{Z}^{2} d-\omega_{X}\left(\omega_{X} d-\omega_{Y} c\right)+\dot{\omega}_{Z} c \\
& a_{P_{2 Z}}=a_{P_{0 Z}}+\omega_{X} \omega_{Z} c+\omega_{Y} \omega_{Z} d+\dot{\omega}_{X} d-\dot{\omega}_{Y} c \\
& a_{P_{3 Z}}=a_{P_{0 Z}}+2 \omega_{Y} \omega_{Z} b+2 \dot{\omega}_{Z} b
\end{aligned}
$$

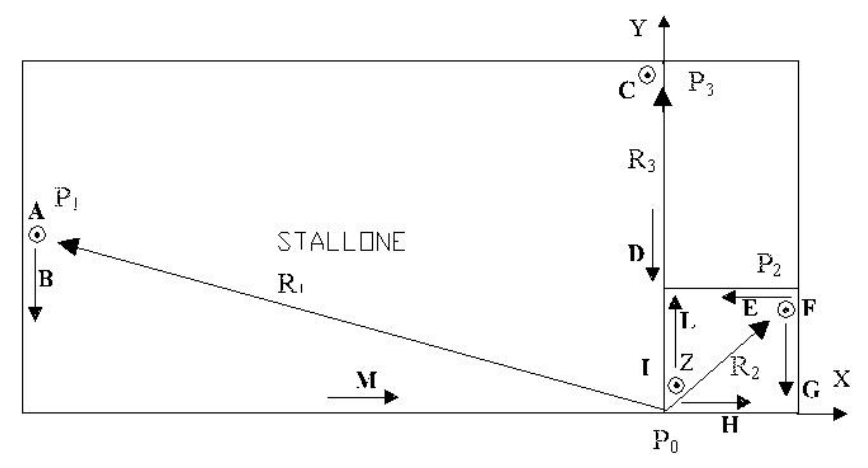

Figure 2. A scheme for earthquake monitoring on the Stallone room at Holy Convent in Assisi (Italy) through installation of seismograph accelerometers at points $\mathrm{P}_{0}, \mathrm{P}_{1}, \mathrm{P}_{2}$, and $\mathrm{P}_{3},[10]$.

where the distances $\mathrm{a}, \mathrm{b}, \mathrm{c}$, and $\mathrm{d}$ are related to the position vectors $R_{1}, R_{2}$ and $R_{3}$ locating point $P_{1}, P_{2}$ and $P_{3}$ respectively with respect to $\mathrm{P}_{0}-\mathrm{XYZ}$.

In the monitoring system of Fig. 2 the seismograph accelerometer outputs have been named as A, B, M, G, F, C, $\mathrm{L}, \mathrm{I}$, and they correspond to the acceleration components $\mathrm{a}_{\mathrm{P} 1 \mathrm{Z}}$, $\mathrm{a}_{\mathrm{P} 1 \mathrm{Y}}, \mathrm{a}_{\mathrm{P} 2 Z}, \mathrm{a}_{\mathrm{P} 2 \mathrm{Y}}, \mathrm{a}_{\mathrm{P} 3 \mathrm{Z}}, \mathrm{a}_{\mathrm{P} 1 \mathrm{X}}, \mathrm{a}_{\mathrm{P} 0 \mathrm{X}}, \mathrm{a}_{\mathrm{P} 0 \mathrm{Y}}, \mathrm{a}_{\mathrm{P} 0 \mathrm{Z}}$, that have been used to compute the Kinematics of the angular motion of the floor plane as outlined in Eq. (1).

The system of Eq. (1) is linear with respect to $\dot{\omega}_{\mathrm{x}}, \dot{\omega}_{\mathrm{y}}$, $\dot{\omega}_{\mathrm{z}}$, but quadratic with respect to $\omega_{\mathrm{x}}, \omega_{\mathrm{y}}$ and $\omega_{\mathrm{z}}$. Thus, in order to eliminate the sign ambiguity initial conditions must be taken into account.

After suitable algebraic manipulations and numerical computation the angular motion of the floor plane can be evaluated from the above-mentioned nine acceleration components as shown in the illustrative example of Fig. 3.

The example of Fig. 3 shows a 3D angular motion that can be considered of significant magnitude and therefore may have relevant effects on the dynamics of constructions, as shaking it. In addition, one can observe the typical time history of seismograms, for the generating ones of Fig. 3, with large variations that have been computed as shown in Fig. 3b).

From practical viewpoint considerable problem concerns with the simultaneous and synchronized acquisition by the nine seismograph accelerometers, since the seismic station works automatically in the sense that the acquisition of each accelerometer is started when each accelerometer feels a suitable threshold value. This value is fixed for each accelerometer being of different type and sensitive location. However, for this investigation on numerical computation of seismic angular motion, with the help of Dr. Gorelli of SSN it has been possible to identify suitable acquired data as for the case of Fig. 4.

\section{CAPAMAN DESIGN}

CaPaMan (Cassino Parallel Manipulator) is a 3-DOF spatial parallel manipulator that shows a robust mechanical design [16], [17]. It has been conceived and built at LARM: Laboratory of Robotics and Mechatronics in Cassino, Italy. 

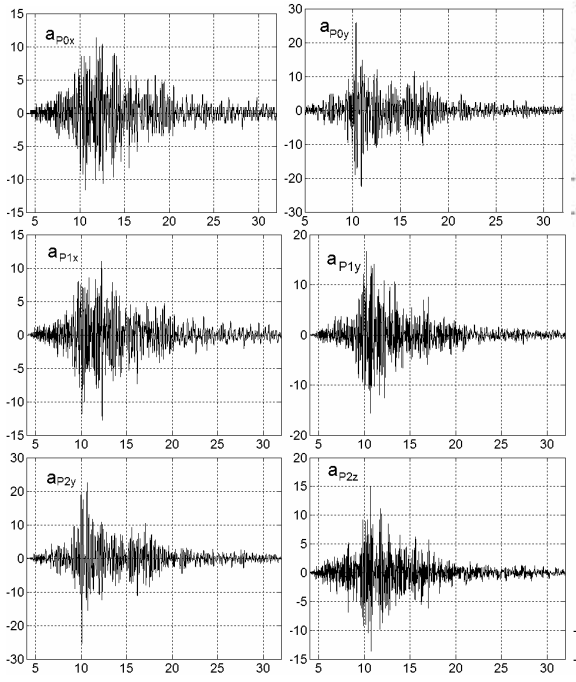

a)

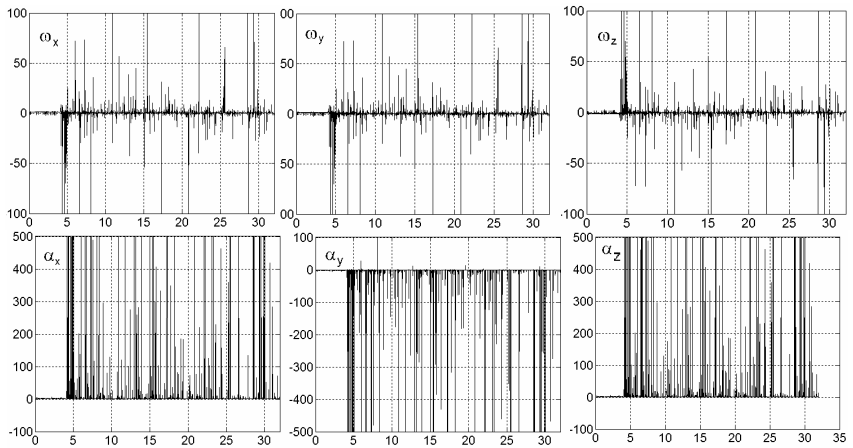

b)

Figure 3. An example of 3D seismic motion for the Stallone room floor at Holy Convent in Assisi (Italy) in Fig. 4 during the earthquake of 14 Oct. '97 at 15.23,42: a) acquired seismograms [10] expressed in $\mathrm{cm} / \mathrm{sec}^{2}$ vs time; b) computed angular velocity and angular acceleration components expressed in $\mathrm{deg} / \mathrm{sec}^{2}$ and $\mathrm{deg} / \mathrm{s}^{2}$ vs time.

CaPaMan is composed by a fixed platform $\mathrm{FP}$ and a moving platform MP, as shown in Fig. 4. MP is driven by 3 legs that are connected to it by spherical joints located at the corresponding articulation points.

This gives to the mechanism the mobility of a 3-DOF spatial mechanism. The legs are composed of an articulated parallelogram AP, a prismatic joint SJ and a connecting bar CB. CB may translate along the passive prismatic joint SJ, keeping its vertical posture.

The input kinematic variables of the robot are $\alpha_{k}$ which are the input crank angles, for $\mathrm{k}=1,2,3$. Moreover $\mathrm{P}_{0}$ and $\mathrm{O}$ denote the center point of MP and FP, respectively.

The application as earthquake simulator is based on the feasibility of easy programming of suitable leg movements that give a seismic motion to the mobile platform.

In addition, CaPaMan prototype has been provided of suitable accelerometers as shown in Fig. 5a) in order to monitor and verify the seismic simulated motion. The built prototype is shown in Fig. 5b).

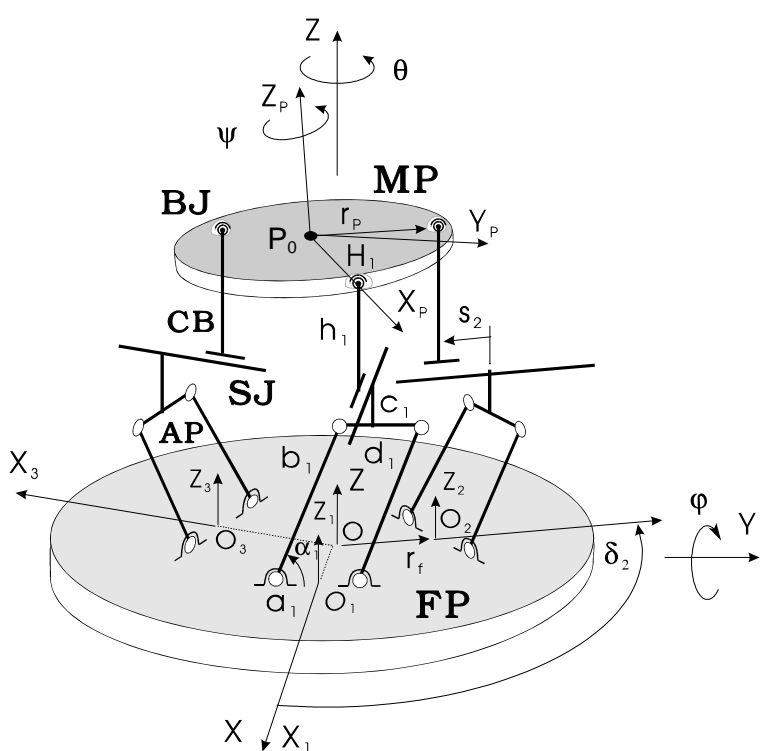

Figure 4. Kinematic chain of the CaPaMan (Cassino Parallel Manipulator) at LARM in Cassino.

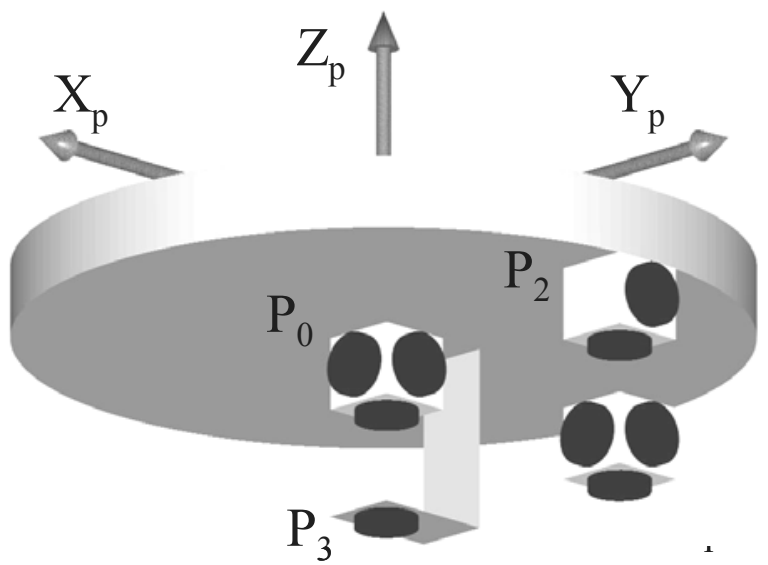

a)

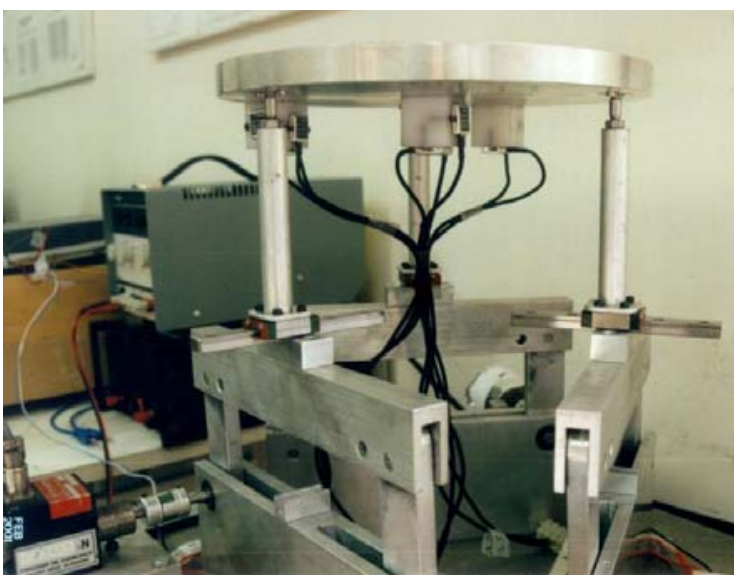

b)

Figure 5. CaPaMan design: a) a scheme of MP for the accelerometers placement on points $\mathrm{P}_{0}, \mathrm{P}_{1} ; \mathrm{P}_{2}$ and $\mathrm{P}_{3} ; \mathrm{b}$ ) prototype with accelerometers and a torsionmeter for an application as sensored earthquake simulator. 
In particular, accelerometers have been installed at points $\mathrm{P}_{0}, \mathrm{P}_{1}, \mathrm{P}_{2}, \mathrm{P}_{3}$ as shown in Fig. 5a) to obtain a solution of Eq. (1) for the angular velocity in the form

$$
\begin{aligned}
& \omega_{X}= \pm \sqrt{\frac{1}{2}\left(\frac{a_{P_{0 Y}}-a_{P_{2 Y}}+a_{P_{0 X}}-a_{P_{1 X}}}{r}+\frac{a_{P_{3 Z}}-a_{p_{0 Z}}}{L}\right)} \\
& \omega_{Y}= \pm \sqrt{\frac{1}{2}\left(\frac{a_{P_{1 X}}-a_{P_{0 X}}-a_{P_{0 Y}}-a_{P_{2 Y}}}{r}+\frac{a_{P_{3 Z}}-a_{P_{0 Z}}}{L}\right)} \\
& \omega_{Z}= \pm \sqrt{\frac{1}{2}\left(\frac{a_{P_{0 Y}}-a_{P_{2 Y}}-a_{P_{0 X}}-a_{P_{1 X}}}{r}-\frac{a_{P_{3 Z}}-a_{P_{0 Z}}}{L}\right)}
\end{aligned}
$$

and for the angular acceleration in the form

$$
\begin{aligned}
\dot{\omega}_{X}= & \frac{a_{P_{2 Z}}-a_{P_{0 Z}}}{r}+ \\
& -\frac{1}{2} \sqrt{\left(\frac{a_{P_{1 X}}-a_{P_{0 X}}}{r}\right)^{2}-\left(\frac{a_{P_{0 Y}}-a_{P_{2 Y}}}{r}-\frac{a_{P_{3 Z}}-a_{P_{0 Z}}}{L}\right)^{2}} \\
\dot{\omega}_{Y}= & \frac{a_{P_{1 Z}}-a_{P_{0 Z}}}{r}+ \\
\dot{\omega}_{Z}= & -\frac{1}{2} \sqrt{\left(\frac{a_{P_{0 Y}}-a_{P_{2 Y}}}{r}\right)^{2}-\left(\frac{a_{P_{0 X}}-a_{P_{1 X}}}{r}-\frac{a_{P_{3 Z}}-a_{P_{0 Z}}}{L}\right)^{2}} \\
& -\frac{1}{2} \sqrt{\left(\frac{a_{P_{3 Z}}-a_{P_{0 Z}}}{r}\right)^{2}-\left(\frac{a_{P_{0 X}}-a_{P_{0 Y}}}{r}-\frac{a_{P_{1 X}}-a_{P_{2 Y}}}{L}\right)^{2}}
\end{aligned}
$$

In Eqs. (2) and (3) $\mathrm{r}$ is the radial distance of $\mathrm{P}_{1}$ and $\mathrm{P}_{2}$ from $\mathrm{P}_{0}$ and $\mathrm{L}$ is the distance of $\mathrm{P}_{3}$ from $\mathrm{P}_{0}$. The sign ambiguity in Eqs. (2) can be solved by considering initial conditions, i.e. the starting configuration of the MP.

The effects of an earthquake on scaled civil structures have been studied and presented in [18]. For the above-mentioned analysis two more accelerometers have been installed on a scaled civil structure, as shown in the scheme of Fig. 6. In particular, accelerometers have been used to monitor the horizontal and vertical accelerations of a floor point of the scaled civil structure.

Similarly, a torsionmeter has been installed on the shaft of an actuator of the parallel manipulator in order to monitor and verify the feasibility of the actuation, specifically for long events.

The overall test-bed layout is shown in Fig. 6 from which one can appreciate the simplicity of the simulator system, mainly if compared to the current earthquake simulators like those reported in [1]-[7].

The monitoring system is composed of commercial equipment, like an acquisition card NI-ATMIOA16F, [19], industrial accelerometers Kistler 8303A2, [20], and a dynamic torsionmeter FGP CD 1050 [21], that are used in LabVIEW frame [22].
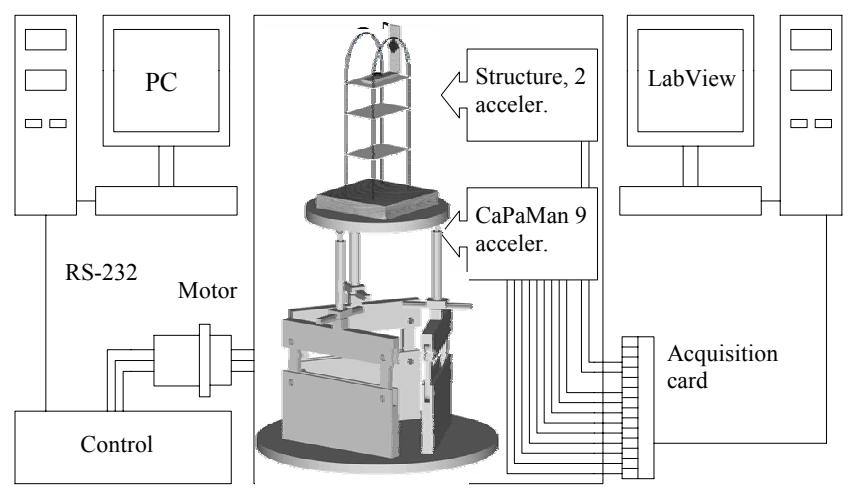

Figure 6. A scheme of sensored CaPaMan as earthquake simulator test-bed.

\section{Programming CaPaMan for EARThQuake SIMULATION}

Frequency, amplitude and acceleration magnitude of a seismic motion can be defined and reproduced by CaPaMan by properly giving the input motion of link $b_{k}(k=1,2,3)$ with a suitable swinging time intervals and ranges for input angles.

When the initial and final positions of MP are assumed as coincident with the rest position of MP, the displacement can be given by the input angles, whose limits are shown in Fig. 7. In particular, $\alpha_{\mathrm{i} 2 \max }$ and $\alpha_{\mathrm{i} 2 \min }$ represent the maximum and minimum input joint angles, corresponding to the maximum and minimum displacements for the seismic motion.

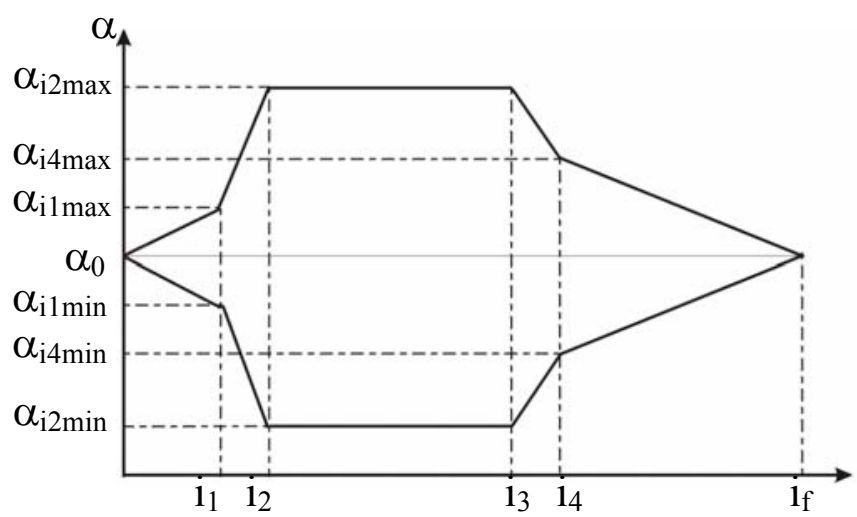

a)

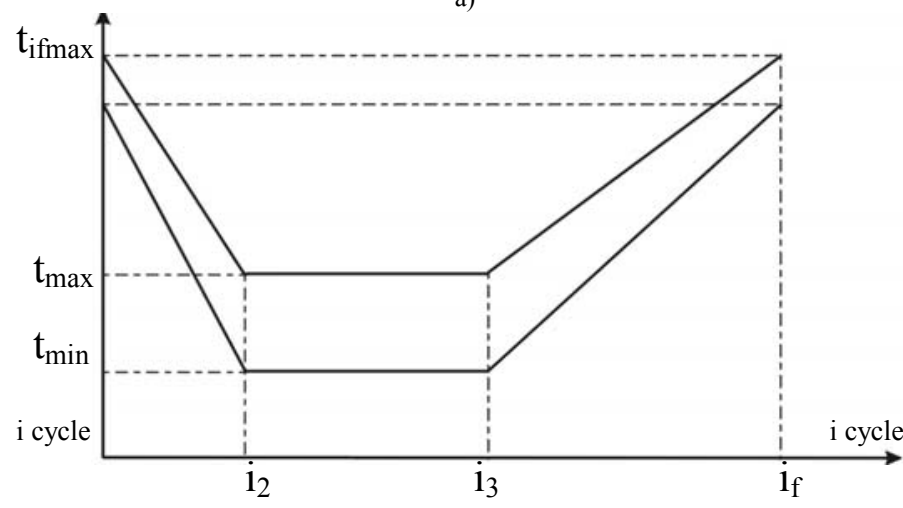

b)

Figure 7. Feasible simulation for earthquake displacements: a) input joint angles as function of number of cycles; b) swinging time duration as function of number of cycles. 
Acceleration peaks can be obtained at the maximum and minimum displacements and they can be computed by using the Kinematics for CaPaMan as formulated in Eqs.(3).

The random characteristics of an earthquake give the possibility to define limits for maximum and minimum accelerations, which can be obtained by limiting the time intervals of each cycle. In particular, a simulated seismic motion can be obtained by giving suitable values for the input joint angles $\alpha_{j}$ and swinging time duration in the form

$$
\begin{aligned}
& \alpha_{j}=\alpha_{j 0}+\left(\alpha_{j i 1 \max }-\alpha_{j i 1 \min }\right) \frac{i}{i_{1}} R+\left(\alpha_{j i 1 m i n}-\alpha_{j 0}\right) \frac{i}{i_{1}} \\
& \mathrm{i} \in\left[0, \mathrm{i}_{1}\right) \\
& \alpha_{\mathrm{j}}=\alpha_{\mathrm{ji} 1 \min }+\left[\left(\alpha_{\mathrm{ji} 2 \max }-\alpha_{\mathrm{ji} 1 \max }-\alpha_{\mathrm{ji} 2 \min }-\alpha_{\mathrm{ji} 1 \min }\right)\left(\frac{\mathrm{i}-\mathrm{i}_{1}}{\mathrm{i}_{2}-\mathrm{i}_{1}}\right)\right] \mathrm{R}+ \\
& +\mathrm{R}\left(+\alpha_{\mathrm{ji} 1 \max }-\alpha_{\mathrm{ji} 1 \min }\right)+\left(\alpha_{\mathrm{ji} 2 \max }-\alpha_{\mathrm{ji} 1 \min }\right)\left(\frac{\mathrm{i}-\mathrm{i}_{1}}{\mathrm{i}_{2}-\mathrm{i}_{1}}\right) \\
& \alpha_{\mathrm{j}}=\alpha_{\mathrm{ji} 2 \min }+\left(\alpha_{\mathrm{ji} 2 \max }+\alpha_{\mathrm{ji} 2 \min }\right) \mathrm{R} \quad \mathrm{i} \in\left[\mathrm{i}_{2}, \mathrm{i}_{3}\right) \\
& \alpha_{\mathrm{j}}=\alpha_{\mathrm{ji} 2 \min }+\left[\left(\alpha_{\mathrm{ji} 4 \max }-\alpha_{\mathrm{ji2max}}-\alpha_{\mathrm{ji} 4 \min }-\alpha_{\mathrm{ji} 2 \min }\right)\left(\frac{\mathrm{i}-\mathrm{i}_{3}}{\mathrm{i}_{4}-\mathrm{i}_{3}}\right)\right] \mathrm{R}+ \\
& +\mathrm{R}\left(+\alpha_{\mathrm{ji} 2 \max }-\alpha_{\mathrm{ji} 2 \min }\right)+\left(\alpha_{\mathrm{ji} 4 \max }-\alpha_{\mathrm{ji} 2 \min }\right)\left(\frac{\mathrm{i}_{4}-\mathrm{i}}{\mathrm{i}_{4}-\mathrm{i}_{3}}\right) \\
& \mathrm{i} \in\left[\mathrm{i}_{3}, \mathrm{i}_{4}\right) \\
& \alpha_{j}=\alpha_{j 0}+\left(\alpha_{j i 4 \max }-\alpha_{j i 4 \min }\right)\left(\frac{i_{f}-i}{i_{f}-i_{4}}\right) R+\left(\alpha_{j i 4 m i n}-\alpha_{j 0}\right)\left(\frac{i_{f}-i}{i_{f}-i_{4}}\right) \\
& \mathrm{i} \in\left[\mathrm{i}_{4}, \mathrm{i}_{\mathrm{f}}\right]
\end{aligned}
$$

By considering Fig.7b) the duration can be evaluated in the form

$$
\begin{aligned}
\mathrm{t}= & \mathrm{t}_{\text {ifmin }}+\left[\left(\mathrm{t}_{\text {max }}-\mathrm{t}_{\text {min }}-\mathrm{t}_{\text {ifmax }}+\mathrm{t}_{\text {ifmin }}\right) \frac{\mathrm{i}}{\mathrm{i}_{2}}\right] \mathrm{R}+ \\
& +\mathrm{R}\left(+\mathrm{t}_{\text {ifmax }}-\mathrm{t}_{\text {ifmin }}\right)+\left(\mathrm{t}_{\text {min }}-\mathrm{t}_{\text {ifmin }}\right) \frac{\mathrm{i}}{\mathrm{i}_{2}} \\
\mathrm{t} & =\mathrm{t}_{\text {min }}+\left(\mathrm{t}_{\text {max }}-\mathrm{t}_{\text {min }}\right) \mathrm{R} \quad \mathrm{i} \in \\
\mathrm{t} & =\mathrm{t}_{\text {min }}+\left[\left(\mathrm{t}_{\text {min }}-\mathrm{t}_{\text {max }}+\mathrm{t}_{\text {ifmax }}-\mathrm{t}_{\text {ifmin }}\right)\left(\frac{\mathrm{i}-\mathrm{i}_{3}}{\mathrm{i}_{\mathrm{f}}-\mathrm{i}_{3}}\right)\right] \mathrm{R}+ \\
& +\mathrm{R}\left(+\mathrm{t}_{\text {max }}-\mathrm{t}_{\text {min }}\right)+\left(\mathrm{t}_{\text {ifmin }}-\mathrm{t}_{\text {min }}\right)\left(\frac{\mathrm{i}_{-} \mathrm{i}_{3}}{\mathrm{i}_{\mathrm{f}}-\mathrm{i}_{3}}\right)
\end{aligned}
$$

in which $\mathrm{R}$ is a random number within the range 0 and 1 . The other angles parameters and durations can be suitably given to simulate a seismogram.

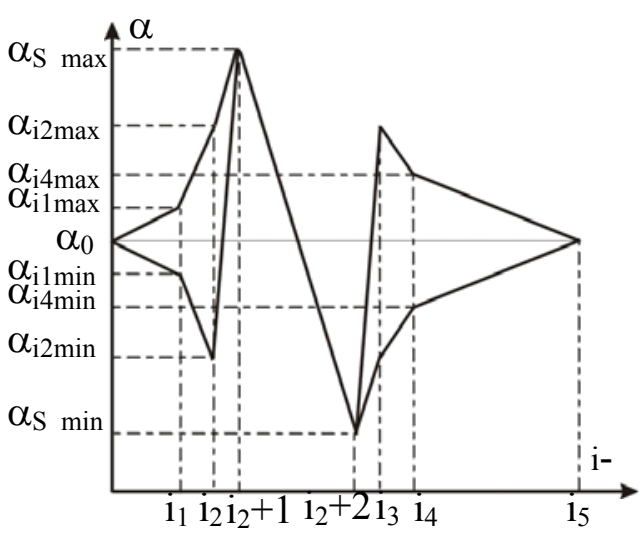

Figure 8. An example for a single wave simulated earthquake.

If one consider as an example a seismograms that is given by a single wave, as shown in Fig.8, Eqs. (4-5) can be simplified in the form

$$
\begin{aligned}
& \alpha_{j}=\alpha_{j S \_m a x} \\
& t=t_{\text {min }} \\
& \alpha_{j}=\alpha_{j S \_m i n} \\
& t=t_{\text {max }}
\end{aligned}
$$$$
\mathrm{i}=\mathrm{i}_{2}+1
$$$$
\mathrm{i}=\mathrm{i}_{2}+2
$$

\section{LABORATORY TESTS}

The prototype of CaPaMan parallel manipulator has been successfully used to simulate earthquake motion characteristics, as reported in previous experiences [11] and [18]. In particular, point seismograms can be reproduced exactly but $3 \mathrm{D}$ seismic motion can be obtained only with similar characteristics of real events.

\section{A. Simulation of Seismograms}

In Figs. 9 and 10 seismograms of horizontal and vertical motions have been simulated. The simulated motions by CaPaMan have been determined by using Eqs. (4) and (5) after suitable numerical elaboration of the data of real seismic events in order to take into account effects of the scaled size through suitable scaled movement displacements, periods, and accelerations.

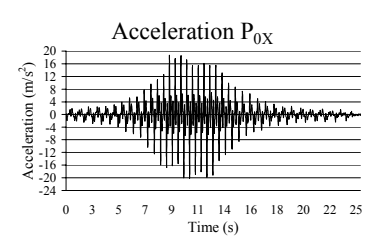

a)

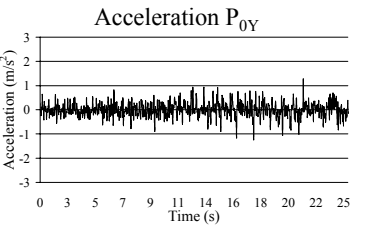

b)

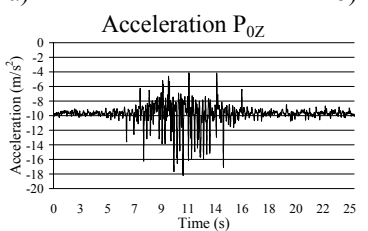

c)

Figure 9. Seismograms of the simulated horizontal earthquake motion by CaPaMan: a) X-component; b) Y- component; c) Z-component. 


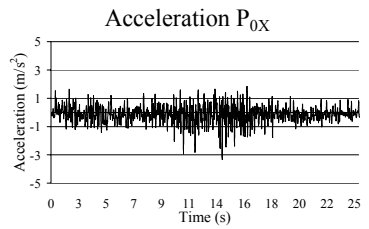

a)

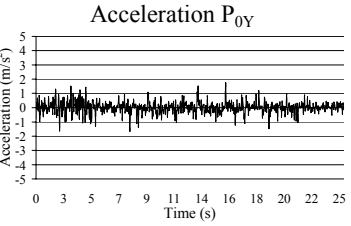

b)

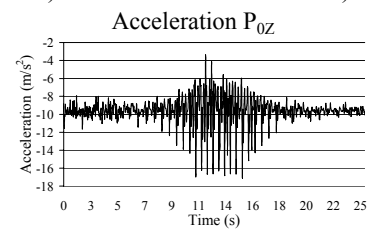

c)

Figure 10. Seismograms of the simulated vertical earthquake motion by CaPaMan: a) X-component; b) Y- component; c) Z-component (vertical direction).

In particular, in Figs. 9 and 10 it is possible to recognize the typical behavior of an earthquake as it is shown in the plots of point seismograms of real earthquakes of Fig. 2 and Fig. 7. Those results show that CaPaMan parallel manipulator can be successfully used as motion simulator and, in particular, as earthquake simulator.

\section{B. Simulation of $3 D$ Motion}

The CaPaMan system has been used to simulate a $3 \mathrm{D}$ motion. In particular, Figs. 11 and 12 show the results of an earthquake motion, which are obtained by CaPaMan.
In Fig. 11 the angular motion characteristics of the MP have been obtained after numerical evaluation through Eqs. (1-3).

In particular, CaPaMan has simulated the $3 \mathrm{D}$ motion of Fig. 5. Acquired data of the nine accelerometers shown in Figs. 5 and 6 are used to compute the rigid body motion of the MP in terms of angular velocity and acceleration, as shown in Figs. 11 and 12.

\section{Earthquake Simulation with a Model for Civil Structures}

Structures in Civil Engineering are subjected to static and dynamical loadings due to wind, earthquakes and traffic loads, which can damage.

Earthquakes simulator experiments allow to model an important class of dynamic loads met in Civil Engineering practice and have proved to be an efficient tool for studying the dynamic response of structures. By considering the effects of seismic motions on these structures, CaPaMan parallel manipulator has been also applied to investigate the effects of an earthquake on a scaled civil structure. In particular, the task is to monitor the effects due to a seismic motion, and compare the acceleration of the MP, which can be considered as the terrain surface, with the acceleration affecting the scaled civil structure.

The system of Figs. 6 and 7 has been used, together with a scaled civil structure, which have been installed on the MP, as shown in Fig. 13. In particular, a scaled civil structure has been considered as composed by wood, to model the floor, and steel, to model the column.
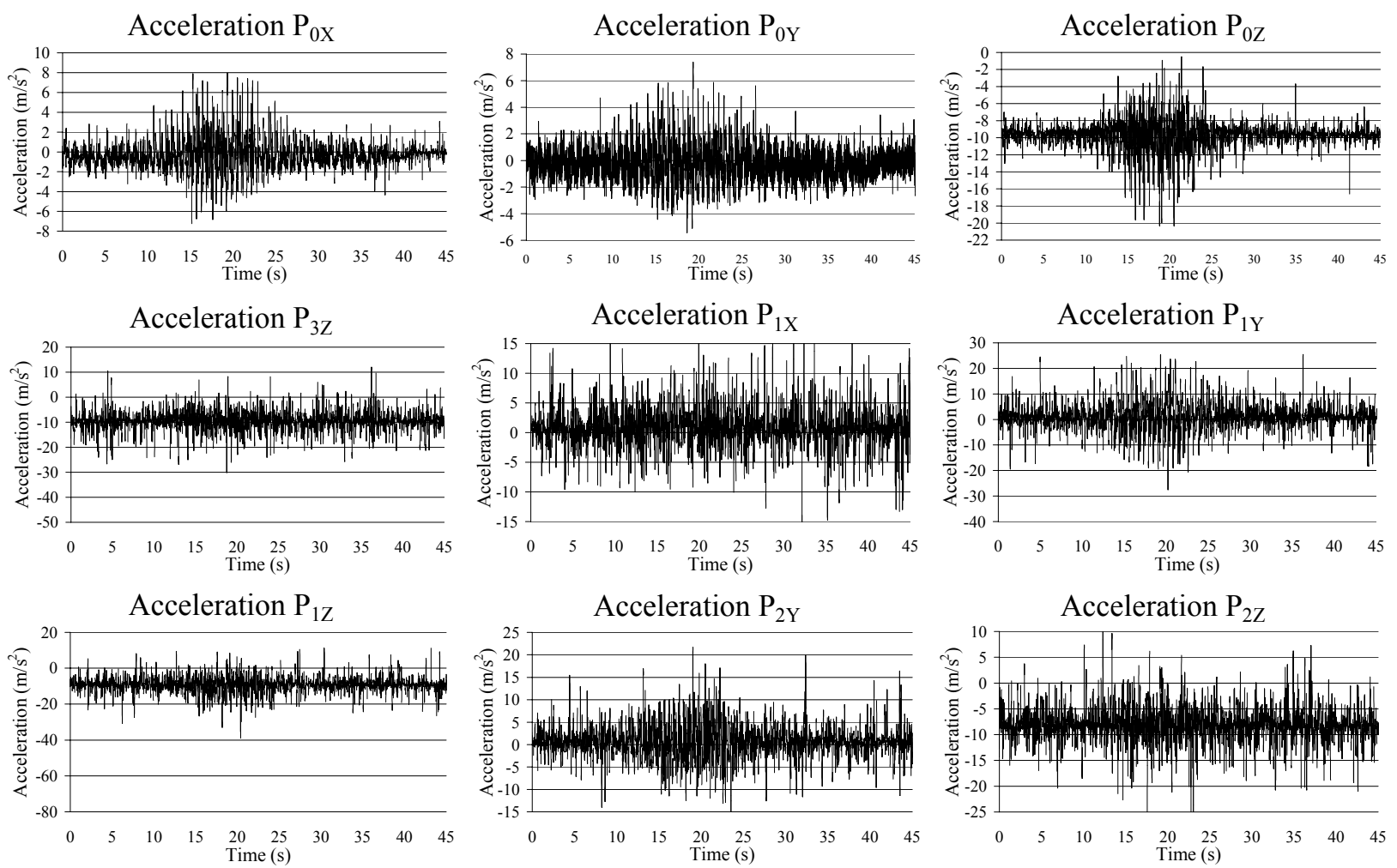

Figure 11. Plots of point seismograms for a simulated 3D seismic motion by CaPaMan manipulator reproducing the case of Fig. 3. 

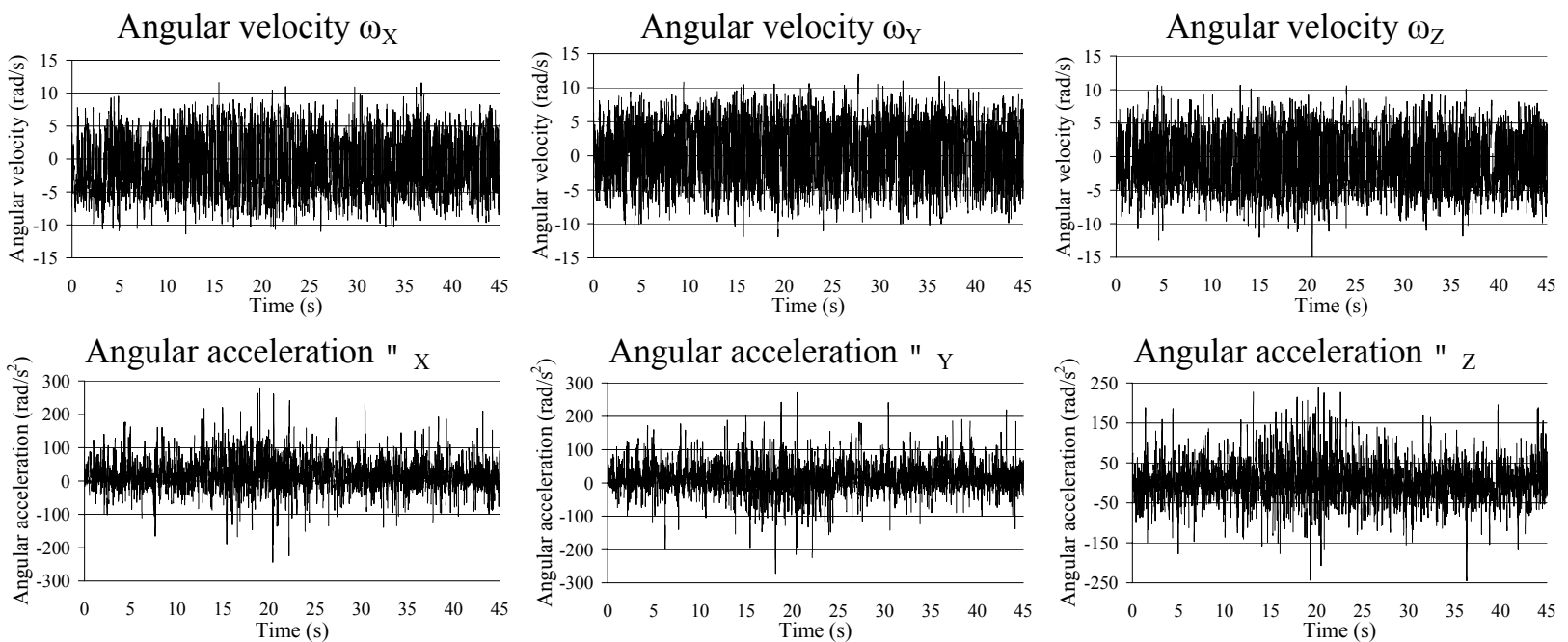

Figure 12. Plots of angular motion characteristics for a simulated 3D seismic motion of Fig. 10 by CaPaMan manipulator reproducing the case of Fig. 3.

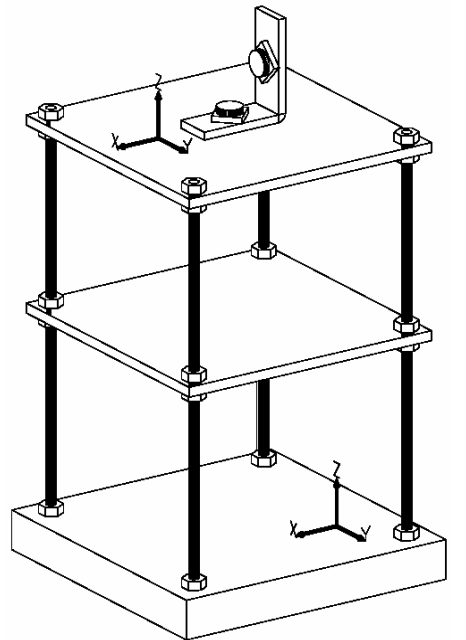

a)

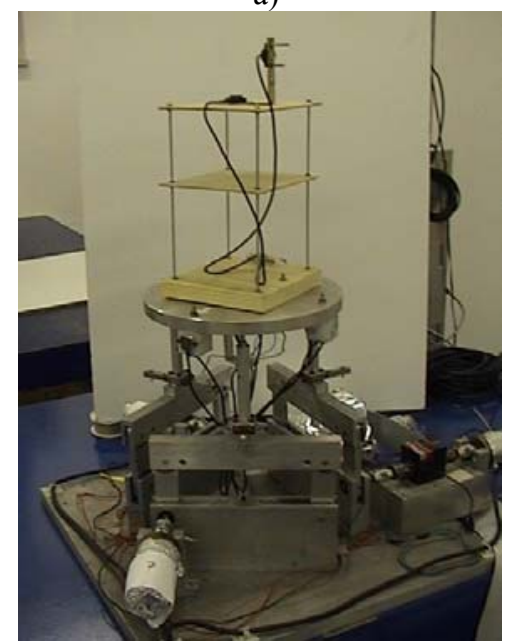

b)

Figure 13. A sensorized scaled civil structure used to monitor the effects of an earthquake simulation: a) a sketch; b) the experimental set-up with the CaPaMan prototype.
It has been suitably built according to the CaPaMan's size in order to not to affect the dynamic behavior of the manipulator and maximize the visual effects of the model's dynamic behaviour. For experimental tests, the scaled civil structure is composed of rigid rectangular plates supported by four columns. The coordinate systems of the structures are shown in Figure 13a).

The $\mathrm{X}$-axis has been chosen along the lateral direction and the $\mathrm{Y}$-axis has been chosen along the longitudinal direction. The columns of the models are assumed to be inextensible and their mass negligible if compared to the mass of the whole system.

Experimental tests of vertical and horizontal earthquakes have been carried out by using CaPaMan and illustratve examples of results are reported in Fig. 14.

In particular, in Fig. 14 a comparison is shown between the point acceleration occurring on the MP and the acceleration occurring on high floor of the scaled civil structure.
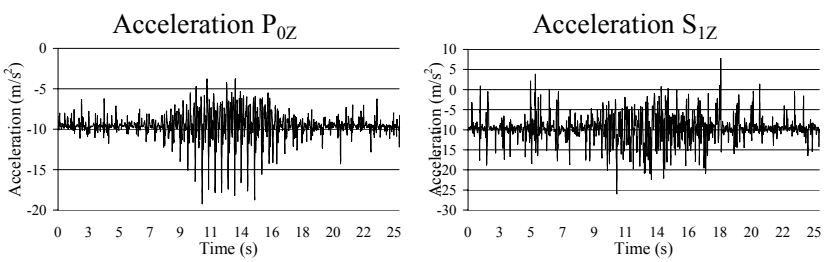

a)
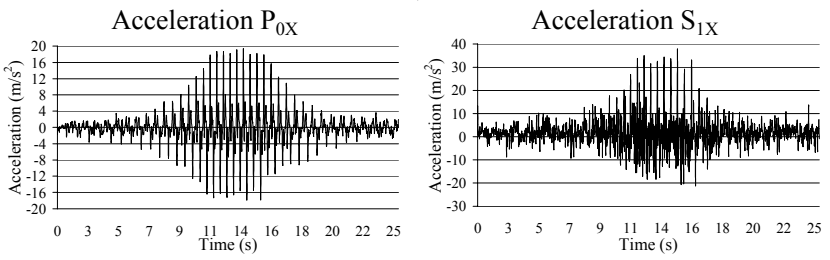

b)

Figure 14. Point seismograms of the simulated earthquake motions by CaPaMan and acquired accelerations $\mathrm{S}_{1 \mathrm{x}}$ and $\mathrm{S}_{1 z}$ on the scaled civil structure : a) vertical earthquake; b) horizontal earthquake. 
As expected, acceleration amplitude of the upper floor of the scaled civil structure is larger than the one occurring at the MP. It is possible also to note a time delay on the plots, this is due to the elastic effects of the scaled civil structure.

\section{CONCLUSION}

A novel application of CaPaMan (Cassino Parallel Manipulator), a 3-DOF spatial parallel manipulator, as earthquake test-bed simulator has been successfully experienced by means of experimental tests. The novelty of the proposed application concerns with the consideration of 3D motion aspects in earthquake events and the feasibility of CaPaMan architecture with 3 -DOF only to replicate 3D seismic motion characteristics. Experimental validation of the $\mathrm{CaPaMan}$ has been presented by monitoring the effect of $3 \mathrm{D}$ motion on scaled structures of civil constructions, according to the size of the CaPaMan prototype.

\section{REFERENCES}

[1] Kajima, "Laboratories \& Facilities", Shaking Table Laboratory, Tokyo, www.kajima.co.jp/tech/katri/leaf/ conte/leaf-e-002.html, 2003.

[2] ISMES, "The Six Degrees of Freedom Shaking Table, MASTER”, Enel Hydro B.U. ISMES, Seriate, 2001.

[3] Structural Dynamics Control \& Earthquake Engineering Laboratory, "Earthquake Simulator Facilities", Department of Civil Engineering and Geological Sciences, University of Notre Dame, Notre Dame, http://www.nd.edu/ quake/facilities/, 2003.

[4] Structural Engineering Group, "Tri-axial Earthquake and Shock Simulator (TESS)", U. S. Army Construction Engineering Research Laboratories, www.cecer.army.mil/fl/seg/tess.htm, 2003.

[5] VCE (Vienna Consulting Engineers), "Shaking Table Test at ISMES Institute", www.vce.at/pages /int_indhtm?/pages/inter/ital.htm, 2003.

[6] Washington University, "Laboratory Facilities", Washington, http://wusceel.civewustl.edu/quake/ facilities.htm.

[7] National Information Service for Earthquake Engineering, "Earthquake Simulator Laboratory", University of California, Berkeley, http://peer.berkley.edu/lab/, 2003

[8] SSN National Seismic Service, "Seismic behaviour of the Holy Convent in Assisi ,Pope Palace, 1995/1998”, CDROM, Rome, 1999. (in Italian)

[9] SSN National Seismic Service, "Elaboration of the Main Acquisition for Earthquakes in the Umbro-Marche Region on Sept/Oct. 1997”, CD-ROM, Rome, 1998. (in Italian)

[10] Pietramellara C. et al., "The Holy Convent in Assisi", Laterza Ed., Bari, 1988. (in Italian)

[11] Ceccarelli M., Ottaviano E., Galvagno M., "A 3-DOF Parallel Manipulator as Earthquake Motion Simulator", Proc. of the 7th Int. Conf. on Control, Automation, Robotics and Vision ICARCV 2002, Singapore, 2002, pp. 944-949.

[12] Ceccarelli M., Carvalho J.M.C., Lanni C., Pugliese F.,
"CaPaMan (Cassino Parallel Manipulator) as Sensored Earthquake Simulator", Int. Conf. on Intelligent Robots and Systems IROS'99, Kyongiu, Vol. 3, 1999, pp. 15011506.

[13] Lanni C. Pugliese F., Ceccarelli M., "Experimental validation of CaPaMan as Earthquake Simulator", IEEE/ASME Int. Conf. on Advanced Intelligent Mechatronics AIM'01, Como, CD Proc., 2001, pp. 153158.

[14]Braga F., et al., "Fundamentals of Antiseismic Engineering”, CISM Ed., Udine,1977, (in Italian)

[15] Symon K. R., "Mechanics", Addison-Wesley Publ. Company, New York, 1989.

[16] Ceccarelli M., "A new 3 D.O.F. Spatial Parallel Mechanism", Jnl. of Mechanism and Machine Theory, Vol. 32, No. 8, pp. 895-902, 1997.

[17] Ceccarelli M., Carvalho J. C. M., “A Closed-Form Formulation for the Inverse Dynamics of a Cassino Parallel Manipulator", Multibody System Dynamics, Vol. $\mathrm{n}^{\circ} 5$, 2001, pp 185-210.

[18] Ottaviano E., Ceccarelli M., Castelli G., "Experimental Results of a 3-DOF Parallel Manipulator a an Earthquake Motion Simulator", ASME Design Eng. Tech. Conf. and Computers in Eng. Conf., Salt Lake City, Paper DETC2004-57075, 2004.

[19] National Instruments, "DAQ Acquisition board AT-MOI16F-5”, General Catalogue, 1994.

[20]Kistler, "Technical data for the accelerometer model 8303A", 1997.

[21]FGP Instrumentation, “ Technical data for the torsionmeter model CD 1050", 1997.

[22] National Instruments, "Software LabVIEW for Windows", 1994. 